\title{
Brain adaptation and alternative developmental trajectories
}

\author{
MARK H. JOHNSON, EMILY J. H. JONES, AND TEODORA GLIGA \\ Birkbeck, University of London
}

\begin{abstract}
Resilience and adaptation in the face of early genetic or environmental risk has become a major interest in child psychiatry over recent years. However, we still remain far from an understanding of how developing human brains as a whole adapt to the diffuse and widespread atypical synaptic function that may be characteristic of some common developmental disorders. The first part of this paper discusses four types of whole-brain adaptation in the face of early risk: redundancy, reorganization, niche construction, and adjustment of developmental rate. The second part of the paper applies these adaptation processes specifically to autism. We speculate that key features of autism may be the end result of processes of early brain adaptation, rather than the direct consequences of ongoing neural pathology.
\end{abstract}

The brain is an organ of adaptation at multiple time scales. Over an evolutionary time scale, the human brain has become adapted to construct and occupy the niche of our species (phylogenetic adaptation). Over the course of ontogeny (individual development), an individual's brain adapts both to the general features of their environment shared with others and to the individual circumstances into which they are born (ontogenetic adaptation). Next, on the time scale of days and hours, human brains can retain survival-relevant information through processes of learning, memory, and attention. Finally, at the scale of milliseconds, our brain adapts to the current sensory input and changes internal states, or prepares motor responses. At these different scales, different complex and dynamic process underlie processes of adaptation. Of particular relevance to child psychiatry is ontogenetic adaptation, how the individual brain adapts in the face of both environmental and intrinsic insults, and issues of how these processes relate to common developmental disorders such as autism and attention-deficit/hyperactivity disorder (ADHD).

Ontogenetic brain adaptation in response to early environmental adversity is related to "resilience." One common view of resilience can be summarized as "the ability of an organism to withstand environmental challenges to normal function" (Karatoreos \& McEwen, 2013). Resilience is typically

The authors acknowledge core funding from the UK Medical Research Council, the Simons Foundation Autism Research Initiative, and the Innovative Medicines Initiative joint undertaking under Agreement 115300, resources of which are composed of financial contribution from the European Union's Seventh Framework Programme (FP7/2009-2013) and EFPIA companies in kind contribution.

Address correspondence and reprint requests to: Mark H. Johnson, Centre for Brain and Cognitive Development, School of Psychology, Birkbeck, University of London, Henry Wellcome Building, Malet Street, London WC1E 7HX, UK; E-mail: mark.johnson@bbk.ac.uk. defined in terms of the extent to which an individual withstands or recovers from early disturbance of their developmental trajectory to achieve normality, and the process is analyzed in terms of relevant genetic and external environmental factors (Cicchetti, 2013; Cicchetti \& Curtis, 2007; Masten, 2007). In contrast, ontogentic adaptation refers to a broader class of processes in which a given individual's brain maximizes its fit to the environment in ways that may not necessarily result in normalization. Further, while in cases of early abuse or neglect the environment is often considered an external variable, it is important to recognize that it is the child's experience of the environment that is critical. Thus, in developmental disorders, factors intrinsic to the brain itself that compromise the child's ability to process the environment may affect the outcome of the optimization process. In contrast to the sustained interest in resilience and adaptation in the face of environmental insults (Cicchetti, 2013; Cicchetti \& Curtis, 2007; Finlay, Yost, \& Cheung, 2006; Luthar \& Cicchetti, 2000), adaptation is rarely considered in the developmental disorders literature. The current neglect of this topic is surprising given the widely held view that common developmental disorders involve widespread disturbances in synaptic function or efficiency (Penzes, Cahill, Jones, VanLeeuwen, \& Woolfrey, 2011; Zoghbi, 2003) that are subject to strong homeostatic pressure (Turrigiano, 2011) and lead, without doubt, to adaptive changes. Despite the brain being the primary organ of integration between genes and environment, we still know very little about the neural basis of resilience and adaptation (Cicchetti \& Curtis, 2007; Curtis \& Cicchetti, 2003). Thus, in the first part of the present paper we focus on the relatively unexplored whole-brain (neural systems) processes and circuits that may protect or buffer individual development in the face of subtle widespread impairments in neural processing that would otherwise threaten typical ontogenetic adaptation. 
In focusing on the active mechanisms of brain adjustment in response to nonoptimal early functioning, the hypothesis arises that the symptoms characteristic of some developmental disorders in midchildhood may be the result of these developmental self-organizing processes, rather than the proximal manifestation of concurrent brain deficits, in a similar way in which the increase in skin temperature accompanying viral infections is not a direct consequence of the virus's presence in the organism but an adaptive response leading to its removal. Focusing on the particular case, we propose that when studying the brain basis of autism in adults, we might in effect be witnessing the end result of a natural alternative trajectory of human brain development. In primarily focusing on brain systems, we do not intend to underplay the importance of genetic or environmental factors and the need for a multilevel approach (Cicchetti \& Curtis, 2007), but merely to place the brain firmly on center stage as the primary organ within which genes and environment interact to ensure optimal functioning of the organism.

\section{Brain Development as a Self-Organizing System}

In many simpler organisms development proceeds through cell lineages that are largely independent of each other, a process described as "mosaic" (Elman, 1998). In mosaic development, a cell's developmental lineage is largely uninfluenced by its immediate biochemical environment or by neighboring cells. In contrast, vertebrate brain development is better characterized as a "regulatory" developmental process in which the division of cells to yield more specialized descendants is dependent on transient changes in their environment (i.e., signals) and on interactions with other cells of similar or dissimilar types. These contrasting developmental mechanisms at the cellular level also apply to the development of regions of the brain in humans. For example, while functional imaging studies in human adults were previously dominated by the search for the specific cortical modules as the home for particular perceptual, cognitive, or linguistic functions, over the past decade the view has emerged that the response properties of a particular brain region are largely determined by its patterns of connectivity to other regions and their current activity states (Friston \& Price, 2001). These and other considerations have led to a domaingeneral framework for studying human functional brain development (interactive specialization) based on the assumption that interactions between brain regions are critical for the development of each one and that networks of regions give rise to emerging functions as a coherent whole (Johnson, 2001, 2011a).

In addition to considering the typical and atypical development of the brain in terms of a whole system, and not as fragmentary parts, brain development is a buffered self-organizing process that leads to an increase in specialization, alongside a corresponding reduction in plasticity (sometimes termed "restriction of fate"). In other words, as development unfolds, increasingly more complex and specialized types of cellular and multicellular structures emerge. In the typical developmental pathway of the human brain, plasticity is reduced and specialization constructed in a way that maximizes the match between the end state behavioral phenotype and our typical social and physical environment. This process is buffered or canalized such that minor perturbations are compensated for. At the genetic and molecular level, we are only just beginning to understand these processes that ensure stabilization. For example, while there are complex dynamics of gene expression derived from developing and adult postmortem human brains with $90 \%$ of the brain-related genes being differentially regulated across either different brain regions or points in developmental time, the vast majority of this differential expression occurs during prenatal development, with patterns of expression tending to become more fixed with increasing age (Kang et al., 2011). Underlying this increasing consistency of gene expression, networks of genes that ensure adherence to the typical developmental pathway through positive and negative feedback processes are being described (e.g., in the fruit fly; Gavin-Smyth, Wang, Butler, \& Ferguson, 2013). Neural system-level factors, which ensure stability of the typical trajectory, are the topic of later sections.

\section{Neural Systems Mechanisms of Ontogenetic Adaptation}

Before moving on to discussing possible neural adaptation mechanisms, some clarification is needed with respect to the use of the term adaptation. When used in the context of evolution and selection, adaptation implies increased fitness, manifested as increased survival rate or reproductive success. This view was also taken with respect to resilience in the face of environmental disruptions (e.g., adaptive changes to a disruptive emotional environment, in the case of maltreated children, protects them from developing psychiatric conditions later in life). However, adaptation will not necessarily produce a "typical" or apparently advantageous phenotype for every individual. For example, ontogenetic adaptation processes chiefly operate early in development, and thus changes in the experienced environment over time can result in later deleterious environment-phenotype mismatches. A classic example is that of fetal malnutrition, thought to trigger the development of metabolic processes designed to retain and store insulin and fatty acids (Barker, 1994). When the individual encounters plentiful food later in life, individuals are at increased risk for health problems that are not seen if the postnatal environment continues to lack resources (Stanner \& Yudkin, 2001). Another example is that of "plasticity" genes like the seven-repeat allele of dopamine receptor D4 (DRD4), which predict greater sociability for children who experience positive parenting, but less sociability for those with unusually bad experiences early in life (Knafo, Israel, \& Ebstein, 2011). Provided an individual's environment is constant (good or bad), the adaptive mechanism will be useful and maintained in the population. Further, neural adaptation is likely to engage processes of homeostasis (the maintaining 
of stability) at multiple levels, from gene expression, to molecular and cellular, and whole system (multicellular) dynamics, including vascular and energetic constraints. In sum, adaptive changes will not necessarily produce behaviors or neural functioning that is "typical"; rather they should produce behaviors and neural functioning that is optimized for that individual's early experience of the environment.

While mechanisms of canalization and plasticity of the developing brain can be studied at different levels from genetic, to molecular, to cellular, to multicellular, we focus on this latter level in which the large networks of billions of neurons show coherent activity, which in turn supports the perceptual and cognitive functions that can go awry in common developmental disorders. Two reasons for focusing on this level of description of the nervous system are as follows: to fully understand processes of ontogenetic adaptation, we need to consider the whole brain, where evidence suggests that some neural systems and regions can compensate for poor functioning in others; and common developmental disorders are associated with widespread changes in the functioning of large-scale neural networks, although these will have multiple underlying molecular and cellular correlates (Johnson, in press).

Four types of whole-brain adaptation merit discussion. First, redundancy is the existence of duplicated functions or neural systems that can compensate for the loss of another under most circumstances. Second, reorganization is the reallocation of functions to regions or networks as orchestrated by critical hubs. Third, niche construction involves changes of neural pathways responsible for selecting the appropriate information from the environment, in accord with not only what an organism needs but also what it can best process. Fourth, timing of developmental trajectories compensates for poor sampling of information from the early environment.

\section{Redundancy}

It is a feature of higher vertebrate brains that there are often multiple neural pathways that can support a given behavior, and thus some in-built redundancy. For example, there are four partially independent neural pathways known to control saccades in primates (Johnson, 1990). These pathways may even compete for control over the same behavioral output (such as simple saccades) in different contexts. Damage to one pathway may result in no apparent change to behavior, except when tested under unusual conditions created in the laboratory. It is possible that this duplication of functionality reflects our evolutionary history, in which adaptive responses were required even when these brains were more primitive in their construction in our evolutionary ancestors. Further, to a limited extent, ontogeny may recapitulate phylogeny with increasingly complex and flexible neural pathways being added to more inflexible automatic routes during individual development (e.g., Johnson 1990).

Following an established computational principle, it seems likely that new brain systems evolve to be complementary to, rather than wholly duplicative of, existing systems
(McClelland, McNaughton, \& O'Reilly, 1995). This raises the seeming paradox of why apparently duplicative pathways should exist in the human brain. Part of the answer to this question may lie in the fact that the brain has not evolved with additions of newly intact functional systems, but rather with differential increases in the volume of whole structures, such as the cerebral cortex (e.g., Finlay \& Darlington, 1995). As new tissue becomes available, gene duplication increases the number of pathways necessary to connect larger amounts of brain volume. However, and in partial agreement with McClelland et al. (1995), duplication was often accompanied by subsequent variation leading to similar but not identical neural pathways (while one gene/pathway continued to function as before, the others were free to adopt new functions or respond to different contexts; Finlay et al., 2006). Various combinations of these duplicated systems may in addition offer more optimally adaptive responses in a wider variety of different contexts and task demands.

The above points are well illustrated in the example of face processing. In addition to a putative subcortical route (Johnson, 2005), there are a number of different regions in the human cerebral cortex known to be involved in the processing of faces ("core face network"), and there are other regions that are more occasionally involved depending on the context ("extended face network"; Haxby, Hoffman, \& Ida Gobbini, 2000). Some of these regions may be more focused on the configural processing of faces (i.e., processing the relationships between facial features), while others are more engaged by featural processing (Cohen Kadosh, Henson, Cohen Kadosh, Johnson, \& Dick, 2010). In neurotypical adults, the patterns of connectivity and activation of these regions depends not only on the presence of faces in the visual field but also on the specific task the participant is engaged in (e.g., identity recognition, gaze direction, or emotional expression; Cohen-Kadosh et al., 2010). For some tasks, a configural processing approach is superior, while for other tasks, a featural analysis of the face may yield more relevant information.

In studying this flexibility of processing, it is evident that successful performance in many face judgment tasks could be achieved in different ways through different routes of cortical processing (redundancy). We know this is the case because general face processing strategies, and the associated patterns of cortical activation, can vary between "neurotypical" adults from different genders or cultures (Chiao \& Ambady, 2007; Li, Yang, Scherf, \& Li, 2013). To understand the potential relevance to developmental disorders, it is worth considering this flexibility of allocation of neural resources in typical development. Cohen-Kadosh et al. (2010) conducted a functional magnetic resonance imaging (fMRI) study with an identical series of face-processing tasks on several different age groups. While regions of the core face network responded to faces from the youngest ages tested, the authors did not observe the task-specific variations in activation seen in adults. In other words, the same cortical network was applied in children regardless of the specific task demands, indicating less flexibility in the processing of faces. It is interesting that 
this lack of task-specific modulation only affected levels of accuracy in some tasks and not others (in this case, while gaze judgments were poorer in children, emotion and identity recognition tasks were not). Thus, in less challenging tasks, success can be achieved with different patterns of neural activation, a point that has relevance to developmental psychopathology (Karmiloff-Smith et al., 2004; Karmiloff-Smith, Casey, Massand, Tomalksi, \& Thomas, 2014).

\section{Reorganization}

It is a fundamental feature of vertebrate brains that they are organized in a hierarchical manner, with some regions or systems exerting modulatory control over others. For example, in saccadic control, a cortical frontal eye field route regulates tonic inhibition of subcortical routes (Schiller, 1985). During human postnatal development, evidence suggests that the brain becomes increasingly hierarchical in its organization (Supekar et al., 2013), though even at birth certain cortical "hub" regions may regulate the activity of other pathways or establish oscillatory activity at specific frequencies (Doria et al., 2010). Reorganization of whole-brain functioning in typical development may occur when new levels of hierarchical control are established. These natural processes of reorganization are also opportunities for the brain to flexibly adapt to minor deviations from the typical intrinsic functioning of regions, or to unusual environments.

At the most basic level of vertebrate brain design, the cerebral cortex exerts a large degree of modulatory control over the more evolutionarily ancient subcortical structures. To a large extent, the cerebral cortex is the primary organ in which genetics and experience combine to result in maximally adaptive behavior. The cortex has even been described as nature's way of escaping genetics. At least within the mammalian line of evolution, there is increasing specialization of regions within the cerebral cortex and clear evidence of a hierarchical organization, although some of these emerge during postnatal development.

From fMRI resting-state analyses and stimulus-activation studies one of the first active hub regions in the human brain is the prefrontal cortex (PFC; Fransson, Aden, Blennow, \& Lgercrantz, 2011). The traditional view of the development of the PFC is that it is the slowest developing region of the human brain, and therefore it was assumed to be the last to "mature." In typical human development, the PFC shows prolonged anatomical development, such as changes in gray matter volume, white matter volume, and cortical thickness throughout childhood and into the teenage years (Gogtay et al., 2004). However, while the structural development of the PFC continues late into postnatal life, there is increasing evidence that it can be functionally activated and is involved in constructing and regulating behavioral responses during early infancy (Grossmann, 2013).

In terms of influencing behavior, the PFC is commonly associated with "executive functions" (Alvarez \& Emory, 2006). As the name implies, this collection of cognitive and behavioral traits is characterized by a brain region that has a controlling influence over other regions, such as inhibition, forward planning, and adapting online to current task demands. While this association between structure and function is widely accepted, the potential role of the PFC in organizing other cortical regions during the course of development remains more speculative. Nevertheless, over the past 20 years, this idea has recurred in the literature (Johnson, 2011a; Thatcher, 1992), raising the further questions of why the PFC is uniquely placed to orchestrate other cortical regions, how it influences these regions, and what role it plays in ontogenetic adaptation?

We start with the first of these questions: why is the PFC uniquely placed to influence other brain regions? There are two reasons why this is the case. The first reason has to do with the PFC initially developing independently of sensory input, and the second reason has to do with its role as a major hub of brain connectivity. While the genetic, molecular, cellular, and computational processes that underlie the parcellation of the cerebral cortex into structural and functional areas remains a topic of active research, an important and hitherto neglected factor may be differences in the timing of neurodevelopmental events between areas of cortical tissue (Cahalane et al., 2011). During prenatal development, projections from the thalamus enter the developing cortex to "capture" their target cortical areas, with perhaps the clearest example in primates being the lateral geniculate nucleus inputs into the primary visual cortex. The sensory input or motor output via the thalamus entrains the still developing cortical neuronal morphology and connectivity in order to fine-tune it for processing specific kinds of information (Cahalane et al., 2011; Sur \& Leamey, 2001). It is no surprise, therefore, that in mammals primary sensory thalamic afferents always innervate the cortex first to form primary sensory areas of cortex. Less obvious is that this approximately posterior to anterior innervation by thalamic afferents runs counter to the timetable of development of the cortical neurons themselves, as cortical neurogenesis proceeds in a rostro-caudal progression with frontal areas of cortex being differentiated before more posterior regions (Cahalane et al., 2011). In other words, there are diametrically opposed gradients of development across the cortex that ensures that while the differentiation in some (more posterior) regions is heavily dominated by sensory and motor input, the differentiation of more anterior regions is not so constrained. In these anterior areas, the early morphological development of neurons and their connectivity will be more shaped by their intrinsic (spontaneous) activity and patterns of interconnectivity with other cortical areas. This happy accident of developmental timing may result in an anterior portion of the cortex that is detached from being directly constrained by sensory input, and therefore it becomes generally biased to processing information that needs to be integrated across other regions. This developmental decalage has another effect, that of protecting the PFC from abnormal input that shapes the function of earlier sensory areas. 
How does the PFC come to orchestrate other brain regions? One idea comes from computational modeling of efficient knowledge-gathering neural network systems. A type of computer model known as knowledge-based cascade correlation (Shultz, Rivest, Egri, Thivierge, \& Dandurand, 2007) involves an algorithm and architecture in which one component recruits other previously trained functional networks as units when required to enhance more complex types of learning. Put simply, this kind of network can learn many tasks faster, or learn tasks that other networks cannot, because it can recruit the "knowledge" and computational ability of other self-contained networks when they are needed. Essentially, a hub learns to select appropriately from a library of available computational regions to orchestrate the best combination for the learning problem at hand. While this class of model is not specifically intended to be a detailed model of brain circuits, it has been used to characterize the PFC (Thivierge, Titone, \& Schultz, 2005) and may help explain the emerging interactions between the PFC and other regions of the cortex, in particular those that have been captured by sensory or motor inputs. This type of computational model also offers a natural explanation for why the PFC is active during the acquisition of a new skill in adults, but once the new skill is acquired (with the appropriate combination of other posterior regions already selected), its level of activity decreases. Similar trends can be seen in development, in which a general migration of activity during childhood from anterior to posterior regions is observed for some networks (Johnson et al., 2005).

The independent development of the PFC and sensory areas is also in line with a "small-world" architecture being functionally optimal early in development. Embedded topology of brain networks emerges from two competing factors: a distance penalty based on the cost of maintaining long-range connections and a topological term that favors links between regions sharing similar input (Bullmore \& Sporns, 2012). For example, in response to brain growth (phylogenetically and ontogenetically), which means signals need to travel further and more cortex needs to be connected, a hierarchical structure has evolved, where information is locally processed by a small network of related areas and then transmitted through hubs to remote areas (Bullmore \& Sporns, 2012). Even within a species, a relationship exists between neuronal path lengths and brain function, as measured by IQ, for example (van den Heuvel, Stam, Kahn, \& Hulshoff, 2009). Wiring optimization principles drive changes in network structure during development as well. As the brain grows and expanding myelination allows for faster information transmission (Menon, 2013), a "small-world" architecture, in which major cortical hubs are confined to primary sensory and motor areas, gives way to longer distance connectivity between a few major hubs (Supekar et al., 2013).

In sum, we have argued that the increasingly hierarchical structure of the brain during ontogeny offers opportunities for reorganization, some of which may occur naturally during the course of typical brain development. We highlighted the role of the PFC in this capacity for reorganization, to the ex- tent that it may organize or initiate differential organization of function to compensate for atypical functioning in other brain regions. In addition, adaptive adjustments to patterns of communication between brain regions may be reflected in changes in connectivity.

\section{Niche construction}

A new approach within evolutionary theory is niche construction. This approach emphasizes how many different species actively construct the environment that best suits their evolutionary adaptation (Laland, Odling-Smee, \& Feldman, 2000). For example, beavers construct dams that not only create suitable homes but also result in deep lagoons that encourage proliferation of a whole new ecosystem, including creating a suitable habitat for prey species of fish and amphibians. Of course, humans are niche constructors par excellence (Laland et al., 2000). However, of relevance to the current issues, it has been suggested that the concept of niche construction (phylogenetic adaptation) could be usefully applied to ontogenetic adaptation (Flynn, Laland, Kendal, \& Kendal, 2013; Frankenhuis, Panchanathan, \& Clark Barrett, 2013), an idea that clearly echoes older theorizing in child development (Scarr, 1992; Vygotsky, 1991). This perspective places emphasis on individual differences, and specifically how the child's active construction of his or her own environment is influenced by the child's temperament and developing personality. Niche construction has a complex developmental trajectory; in infancy and early childhood, the development of adaptive skills increases the individual's capacity for niche construction, as children acquire the tools to seek out their chosen experiences. However, there is also likely increasing canalization of niche selection with age as the range of paths open to a child becomes increasingly constrained. Studying developmental trajectories of behaviors associated with niche construction is thus critical to understanding its role in adaptive responses.

What is the relevance of these ideas for developmental psychopathology? To the extent that individuals contribute to their own niche during development, brain circuits that are important for exploring new niches and manipulating the environment to best suit their current cognitive skills will be those that most critically determine the ability to adapt in ontogeny. Damage or inefficiency in these particular brain systems will decrease the ability to regulate and select appropriate environmental input that best matches the capacity of the brain in question. In contrast, intact information-selection systems in the face of damage elsewhere in the brain could result in appropriate ontogenetic adaptive changes to the environment the child selects. As discussed earlier, we refer to maximizing the potential fit between an individual brain and its current capacities, and components of the environment in which it exists. The form that this adaptation takes may not match in nature or scope that seen as a result of the neurotypical trajectory. For example, diffuse damage that differentially affects "social brain" structures may lead to the 
other brain circuits adapting to these circumstances by withdrawing from the social world and directing attention instead to restricted and comprehensible aspects of the physical environment.

The primary brain circuits for exploring and selecting from the environment are those associated with controlling attention, orienting, and exploration. The human brain has several such systems that are dedicated to orienting the senses toward stimuli of interest, maintaining fixation and attention on selected targets, and regulating the overall states of arousal that determine the mode of information gathering (Gottlieb, Oudeyer, Lopes, \& Baranes, 2013). As discussed earlier, while there is a certain degree of redundancy between these systems, particular systems will become best suited to control behavior in certain circumstances.

Later we speculate on the application of niche construction theory to typical and atypical trajectories of human brain development. A buffered self-organizing developmental system such as the human brain is likely to select aspects of the external environment that best suit its own particular processing capacities (Kidd, Piantadosi, \& Aslin, 2012; Rovee-Collier \& Cuevas, 2009). This could drive a switch in the trajectory of development as increasingly atypical aspects of the environment are selected for further processing in brains that may have difficulty processing common features of our species niche.

\section{Adjusting developmental rate}

In the previous section, we discussed how developmental trajectories could be changed by differential selection of aspects of the environment to process and with which to interact. However, it is also likely that the overall rate of brain development in an individual can be adjusted in order to maximize the chances of a fit with the external environment. As discussed earlier, while phylogenetic adaptation concerns the evolution of species, ontogenetic adaptation refers to the development of specific individuals. However, it is important to remember that the timings of developmental trajectories are themselves selected for during evolution. Selection of particular developmental trajectories is often argued to be the primary mechanism for the evolution of brain and behavior (Finlay \& Darlington, 1995; Frankenhuis et al., 2013). More precisely, "[N]atural selection favors developmental systems that tend to construct adult phenotypes that are successful, relative to other variants, at surviving and reproducing" (Frankenhuis et al., 2013, p. 586). Further, species in which there are significant individual differences in developmental trajectories will "hedge their bets" better in the face of varying and unstable environments. Developmental trajectories that are successful in one environment may be less optimal in another. Most flexible of all are species that have delayed an aspect of their development sufficiently for individuals to be able to sample the early environment, learn its characteristics, and then adjust their future trajectory accordingly (see Frankenhuis et al., 2013, for discussion). What are the implications of these considerations for typical and atypical human brain development?

Primates generally have a much more prolonged timetable for their brain development than do other mammals, suggesting that delayed brain development is important for these species. However, we Homo sapiens have taken this to extremis, with our postnatal cortical development extending roughly four times longer than other primates. Finlay and Darlington (1995) have compared measurements of neuroanatomical developmental landmarks among more than one hundred mammalian species and concluded that the order of landmarks of brain development is greatly conserved across a wide range of species. Further, they noticed that, controlling for overall brain and body size, the time course of these landmarks was related to the relative size of structures of the brain in a systematic way. Specifically, disproportionately large growth occurs in the late-generated structures such as the neocortex when the overall timetable is slowed. By their analysis, the structure most likely to differ in size in the relatively slowed neurogenesis of primates is the neocortex. As a next step, the model has been extended to human prenatal development (Clancy, Darlington, \& Finlay, 2000). The model predicts that the more delayed the general time course of brain development in a species, the larger the relative volume of the later developing structures (such as the cerebral cortex, and particularly the frontal cortex) will be. In accordance with this general prediction, the slowed rate of development in humans is associated with a relatively larger volume of cortex and an especially large frontal cortex. Thus, it is likely that the increased extent of the cortex in human brains, and particularly the PFC, is a happy by-product of slowing down the overall timetable of brain development. Additional benefits of the typical trajectory of human brain development relate to the fact that we are born with a very substantial amount of brain development remaining (nearly two decades' worth). This bestows the benefit of substantial environmental influence on brain structure and functional development, and more particularly offers the chance for early sampling of the environment to modulate the overall rate of subsequent development.

From these and other observations, it is clear that a fundamental aspect of human evolution is a greatly slowed trajectory of brain development that extends into two decades of postnatal life. As a successful species, it is likely that there is variation in the trajectory of overall timing of postnatal brain development around an optimal rate for our current most commonly experienced human environments, and having the optimal rate of brain development will be beneficial in the ideal environmental niche for our species. Initial lines of evidence suggest this may be the case.

On consideration, it seems likely that three factors will determine the trajectory of postnatal brain development: variation between individuals of our species (based on genetic variants), adverse early environments that may delay or accelerate the subsequent rates of development, and quality of neural processing of the early environment that could 
have similar consequences to those induced by less predictable external environments.

Taking the first of these factors, very little is currently known about how genetic variation influences overall rates of brain development. A few genes have been identified that modulate the rate of brain growth and whose mutation leads to high functioning microcephaly (meaning that they produce mild phenotypes and prolonged survival; Gilbert, Dobyns, \& Lahn, 2005). These genes have changed dramatically during evolution and are believed to confer adaptive advantages by allowing brain size to fit environmental constraints. Based on theories of the evolution of developmental systems, it seems likely that our highly successful species will contain considerable interindividual variations in overall brain development timing that hedge the necessary bets for the possible variations in the environments we are likely to encounter. A speculation is that more extreme variation (faster or slower), if not lethal, will confer vulnerability to disturbances of the typical developmental trajectory.

Another potential source of variation in the later time course of postnatal development is early sampling of the environment. So-called dynamic optimization modeling of developmental trajectories seeks to link natural environments (evolutionary ecologies), developmental systems, and end-state phenotypic distributions (Frankenhuis \& Panchanathan, 2011). One of the key findings from this work is that early stochastic sampling of the environment can induce individual differences in later developmental plasticity and timing. Individual brains that are initially exposed to a highly predictable early environment and are able to homogenously sample environmental cues can come up with a reliable estimate about their future environment, and therefore tend to specialize relatively early. Other individual brains that have an inconsistent early environment, or who process their environment inconsistently, need to continue sampling their environment by delaying plasticity for as long as possible. As a result, some individual brains will continue sampling their environment (prolonging plasticity), while others can already specialize. Thus, interindividual variation in sensitivity to environmental information at specific points may be the result of the consistency of early experience in a domain.

\section{Autism as a Common Variant Developmental Trajectory}

In this part, we build on the discussion above, but focus on one particular common developmental disorder: autism. It has gradually become apparent that autism is characterized not only by developmental delay but also by atypical developmental trajectories. Recent work has observed atypical trajectories of development from infancy (e.g., Jones \& Klin, 2013), and theoretical perspectives have suggested that autism may be a disorder of sensitive periods (Kroon, Sierksma, \& Meredith, 2013; LeBlanc \& Fagiolini, 2011; Meredith, Dawitz, \& Kramvis, 2012; Thomas, Knowland, \& Karmiloff-
Smith, 2011). However, these hypothesized altered developmental trajectories are rarely thought of as reflecting processes of early compensation in response to an environment that is processed with low fidelity due to increased or decreased levels of neural noise. For example, atypical trajectories of brain overgrowth have been widely noted in autism (e.g., Courchesne et al., 2007; Shen et al., 2013); however, few have considered that this might relate to the prediction that a slowed trajectory of development (a common compensatory response to an inconsistent experience of the environment) will lead to increases in the volume of higher cortical structures during both phylogeny and ontogeny (Clancy et al., 2000). Specifically, we propose that in some cases that lead to autism, synaptic dysfunction leads to the early environment being sampled with poor fidelity, with a particular cost to the most dynamic and least easily predictable elements of the external environment. In many cases, these are associated with the social world and the complex and pseudo-predictable interactions with other humans. A mild degree of noisy sampling may lead to prolonged plasticity and delays in the subsequent steps of brain development that may be specific to the most complex and dynamic aspects of the early environment.

To demonstrate that key aspects of the autism phenotype mainly reflect ontogenetic adaptation, we will initially discuss evidence for early disturbances in synaptic function that would compromise early processing of the external environment, and then discuss preliminary evidence for the mechanisms of ontogenetic adaptation covered above that we believe are at work in shaping the autism phenotype: redundancy, reorganization, (differential) niche construction, and adjustment of the developmental rate.

\section{Synaptic function and autism}

Like other common developmental disorders, autism is both genetically highly heterogeneous and appears to induce multiple and widespread differences across different brain regions and systems. While hundreds of genes have been implicated in autism, many of these are associated with activity-dependent synaptic function (Ebert \& Greenberg, 2013), such as in regulating synapse morphology or levels of specific receptors or transmitters. Even in cases of monogenic causes of autism, such as fragile $\mathrm{X}$ and tuberous sclerosis, knockout mouse models subsequently show developmentally regulated and widespread synaptic consequences (Kroon et al., 2013; Yizhar et al., 2011). While we have good neuropsychological models and scientific strategies for investigating discrete damage to localized regions of the brain, our conceptual understanding of the effects of widespread and diffuse alterations of synaptic function is very poor.

What neural effects would result from early dysfunctions in the genetic pathways that have been identified with autism? Rubenstein and Merzenich (2003) argued cogently that an atypical balance of excitatory and inhibitory activity within brain circuits may be a common consequence of "the co-in- 
heritance of multiple alleles that each contribute to weakening a specific physiological process" (p. 263). In general, the balance of excitation to inhibition in the cortex is regulated by activity levels of glutamatergic and GABAergic neurons, with around $80 \%$ of cortical neurons being excitatory (glutamatergic) pyramidal cells and most of the remainder being inhibitory (GABAergic) interneurons. Several of the mutations linked most closely to autism spectrum disorder (ASD) would likely influence the balance of glutamergic and GABAergic synapses, leading to excitation/inhibition imbalances in the mature brain (e.g., Chih, Engelman, \& Scheiffele, 2005; Graf, Zhang, Jin, Linhoff, \& Craig, 2004; Schroer et al., 1998).

Although much work has focused on characterizing the likely effects of synaptic dysfunction on the mature brain, recent genetic evidence indicates that most genes associated with autism have patterns of peak expression during very early development. For example, Parikshak et al. (2013) observed enrichment for inherited variants associated with autism in networks of genes that start to be upregulated around Postconception Week 22 and are likely involved in synaptic development, and for rare de novo variants in networks that act early in fetal life (Weeks 8 to 22 postconception) and regulate transcription during early neuronal fate determination, migration, and cortical lamination. Further, Willsey et al. (2013) found enrichment of ASD-associated genes in networks with peak expression in the PFC and motor-somatosensory regions during midfetal development (Postconception Weeks 10 to 19). Thus, many genes implicated in risk for autism likely have their most critical effects early in development.

Disruption to the GABAergic or glutamergic systems will likely have different effects in early development than in the mature brain. First, GABA has a largely excitatory function in early postnatal development (Ben-Ari, 2002; Ben-Ari, Khalilov, Kahle, \& Cherubini, 2012). There is an activity-dependent shift to inhibition in early postnatal development that drives the opening and closing of critical periods in sensory cortices (Hensch, 2005; Hensch et al., 1998). Second, imbalances in excitatory and inhibitory activity are likely to be significantly moderated by homeostatic cell mechanisms that maintain network stability in the mature brain (Turrigiano, 2011; Turrigiano \& Nelson, 2004). However, the operation of homeostatic mechanisms varies across development. For example, in the visual cortex, homeostatic compensation turns off at the opening of the critical period in cortical layers that receive primary visual input (Desai, Cudmore, Nelson, \& Turrigiano, 2002; Maffei, Nataraj, Nelson, \& Turrigiano, 2006; Maffei, Nelson, \& Turrigiano, 2004), but turns on in the layers that mediate lateral interactions between different cortical regions (Goel \& Lee, 2007; Maffei \& Turrigiano, 2008). These complexities in developmental timing indicate that deficits in inhibitory/excitatory balance will vary across different periods of development. Third, evidence from mouse models of neurodevelopmental disorders supports the proposal that some synaptic phenotypes are transient in nature (Kroon et al., 2013). For example, in fragile mental re- tardation 1 ( $f m r l)$ knockout mice, altered plasticity in the somatosensory cortex that is observed during the first postnatal week has normalized by the third week (Harlow et al., 2010); this may relate to peak expression of the $f m r l$ gene, which is upregulated between Postnatal Days 4 and 14 (Hoerder-Suabedissen et al., 2013). These earlier transitory atypicalities may have knock-on effects on other aspects of development, which live on as secondary consequences of the initial imbalance. Thus, models of the role of disruptions to the GABAergic and glutamergic systems in ASD must take a developmental perspective.

While the consequences of major imbalances between excitatory and inhibitory processes are understood (e.g., epilepsy), the computational consequences of mild imbalances or dysregulation in early development remain largely unknown. One of the functions of intrinsic inhibitory processing is to increase the signal to noise ratio by "cleaning up" spontaneous neural firing that is not directly linked to stimulus presentation or ongoing processing (Toyoizumi et al., 2013). However, on the flip side, certain levels of background "neural noise" may be critical to the development and specialization of cortical regions. Appropriate levels of noise can ensure that neural networks adaptively settle to appropriate configurations for the data processed, because it ensures that the network is not captured by local minima (Davis \& Plaisted-Grant, in press). However, excessive noise can also mask the appropriate signal, resulting in delayed opening of critical periods (Toyoizumi et al., 2013), delayed specialization of neural networks (Rubenstein \& Merzenich, 2003), and changes in brainwide connectivity (Eichler \& Meyer, 2008).

Any successful account of the "final common pathway" for autism will have to account for why multiple different genetic, molecular, and neural factors can cause the same syndrome (a phenomenon known as "equifinality"). There is now emerging evidence that different genetic pathways might converge on a small number of processes involved in synaptic function and structure (Voineagu et al., 2011). One further contribution to resolving this paradox is if these multiple different synaptic dysfunctions all activate common processes of ontogenetic adaptation during human brain development (e.g., redundancy, reorganization, niche construction and changes in developmental timing). From this perspective, the continuity of the syndrome comes from the brain's typical responses to early widespread synaptic differences, and the syndrome itself is defined and characterized by this adaptive response (as opposed to an ongoing neural deficit). Here, we discuss evidence for the operation of the adaptive processes discussed in the Neural Systems Mechanisms of Ontogenetic Adaptation section in ASD, and their relation to poor fidelity of processing of the early environment.

\section{Redundancy}

We discussed evidence for the existence of redundant cortical systems in the primate brain in the Neural Systems 
Mechanisms of Ontogenetic Adaptation section. In ASD, although early deficits in synaptic functioning are likely widespread, spatiotemporal profiles of gene expression indicate that some areas of the brain are likely more affected than others (Voineagu et al., 2011). Thus, individuals may benefit from using alternate systems to perform similar functions. There are many examples of fMRI studies that report apparently typical behavioral performance despite atypical patterns of brain activation in individuals with ASD. For example, Pierce, Müller, Ambrose, Allen, and Courchesne (2001) tested face perception in adults with autism; despite normal behavioral performance, unlike in neurotypical controls, no activation was seen in the fusiform face area. Autistic adults mainly activated visual areas or the frontal cortex, in an individual specific manner, which suggests that adaptation can lead to different solutions in different individuals. Further examples of atypical brain activations in autism abound. Williams et al. (2006) found typical performance on an imitation task, but significantly different patterns of neural activity, with reduced activity in areas associated with the mirror neuron system in individuals with ASD. The common practice of requiring that groups of participants show similar behavioral performance in neuroimaging studies likely ensures that the majority of group differences observed reflect adaptive use of redundant neural systems. This practice also biases findings toward high-functioning populations (able to follow instructions). It could be that the ability to switch over to alternative pathways is in itself a predictor of functional development. The hypothesis has been made that the severity of an initial brain insult determines when alternative pathways are engaged. Smaller lesions may be insufficient to trigger plasticity and therefore lead, paradoxically, to worse outcomes (Dehaene-Lambertz, Herz-Pannier, \& Dubois, 2006). A greater focus on identifying the systems that are supporting typical behavioral performance in individuals with ASD may be an important step toward developing training programs to help individuals recruit compensatory networks for more difficult tasks.

\section{Reorganization}

In in the Neural Systems Mechanisms of Ontogenetic Adaptation section, we outlined the role of the PFC in adaptive recruitment of alternate brain regions. What role might the PFC play in processes of ontogenetic adaptation in ASD? Autism has been commonly associated with "deficits" in executive functions (EF). While these deficits often co-occur with diagnostic symptoms, evidence indicates that they are dissociable to at least some extent. Thus, Johnson (2012) argues that instead of poor EF skills being part of a core cluster of symptoms of atypicality or impairment in some developmental disorders, good EF skills actually allow the brain to better reorganize in the face of atypical functioning in other regions in individuals at genetic risk. According to this view, poor EF skills are associated with some developmental disorders because these individual children at risk have less capacity to adapt their neural processing in the face of other risk factors early in life. In contrast, children born at risk with strong EF skills have brains that are generally better able to reallocate function from one neural system to another, and thus, on average, tend not to end up with a diagnosis. In other words, being at the lower end of typical variation in EF skills early in life may be considered to be an additional risk factor due to having less capacity to adapt in response to other perturbations to the typical developmental pathway.

Johnson's (2012) argument is based on the findings that while several developmental disorders have been characterized as including EF "deficits," in at least some disorders, these are partially dissociable from other symptoms. For example, while ADHD was traditionally thought of as a disorder primarily affecting prefrontal-striatal systems, and thus primarily affecting $\mathrm{EF}$, it is now recognized to be a more widespread deficit of which EF and the prefrontal systems are only one feature (Castellanos \& Proal, 2012). Similarly, in autism, group differences on EF measures (compared to non-ASD mental age-matched controls) do not imply that all individuals with ASD have EF problems, particularly when considering the milder variants of the condition (Losh et al., 2009; Yerys et al., 2009). Thus, at least for autism and ADHD, poor performance on EF tasks is associated at a group level but may not be a critical core feature of the condition.

Johnson (2012) also points to new evidence that strong EF skills are a protective factor in those born at risk of later emerging developmental disorders because structural and functional MRI studies reveal glimpses of the processes of brain adaptation that may result in typical outcomes from some children born at risk. For example, Pelphrey et al. (2003, cited in Kaiser et al., 2010) studied patterns of brain activation in children with autism, unaffected siblings of children with autism, and controls, using fMRI while they viewed videos of biological motion. Unaffected siblings of children with autism had some patterns of activation in common with those with autism, but not with controls. The authors argued that this "trait" activity is consistent with a "neuroendophenotype" that extends to unaffected family members and raises the possibility that unaffected siblings actively overcome this atypicality in some way. Consistent with this view was the existence of "compensatory activity" patterns of activation, mainly in the superior temporal sulcus and in the PFC, which were not evident in either those with autism or the controls. Thus, these activations had the hallmark of regions whose additional activity allowed children potentially at risk to achieve a typical outcome.

In addition to Johnson (2012), other authors have previously proposed a role for the PFC in compensating for the core deficits in other brain systems that may underlie ADHD (Fassbender \& Schweitzer, 2006; Halperin \& Schulz, 2006). For example, Fassbender and Schweitzer (2006) reviewed a number of neuroimaging studies consistent with the idea that poor PFC (and anterior cingulate) function in those diagnosed with ADHD reduces the individual's ability 
to optimally recruit other brain systems as required for specific cognitive tasks. In other words, while neurotypical individuals (with good PFC function) can flexibly recruit different brain systems and strategies as appropriate for a given task, those with poor PFC functioning (associated with ADHD) are more restricted and limited in their options for neural processing.

Another aspect of neural reorganization that may represent an adaptive change in ASD is neural connectivity. Atypical anatomical and functional connectivity has been identified in many if not all developmental disorders (Menon, 2013). In many cases, these have been described as causal pathways to symptom manifestation (e.g., the decrease in long-distance connectivity and autism; Geschwind \& Levitt, 2007). Reversing this causal relationship we suggest that in many developmental disorders, altered connectivity may actually be a result of brain reorganization to optimize functioning (Liston, Malter Cohen, Teslovich, Levenson, \& Casey, 2011). This was suggested in the case of Alzheimer disease, where the decrease in long-distance connectivity and the increase in clustering augment the efficiency of a brain with low metabolism (Bullmore \& Sporns, 2012). Although for a long time autism was associated with decreased connectivity, especially longdistance connectivity (Geschwind \& Levitt, 2007), recent years have painted a more mixed picture of both decreased and increased connectivity, varying depending on which pathways were the focus of analysis or on the age of the participants (Uddin, Supekar, \& Menon, 2013; Vissers, Cohen, $\&$ Geurts, 2012). Less is known about the very early development of connectivity. However, when structural connectivity was assessed in 6-month-old infants at risk for autism who developed symptoms of this disorder (Wolff et al., 2012), the main finding was of increased connectivity in projection pathways, that is, those pathways connecting frontal and parietal areas with the posterior cortex. By 24 months, though, connectivity levels had decreased below those of controls, which is consistent with adult findings.

There is mounting evidence that connectivity changes could occur as a result of an initial disturbance to excitation/inhibition (E/I) balance (Eichler \& Meyer, 2008). This evidence comes predominantly from work with animal models. For example, mice with a distal-less homeobox 1 (DLXI) deletion restricted to forebrain GABAergic neurons experience aging-related loss of inhibitory interneurons in the cortex and hippocampus during adulthood (Cobos et al., 2005). The resulting reduction in inhibition leads to the withdrawal of glutamate receptors from excitatory neurons in order to maintain homeostatic balance (Howard, Rubenstein, \& Baraban, 2013). As a result, these mice show reduced functional connectivity in the auditory cortex (Seybold et al., 2012), which may relate to recent findings of underconnectivity between language areas in children with autism (Dinstein et al., 2011). Seybold et al. noted that when faced with overactivity, "the nervous system may sacrifice connectivity and computational power for stability" (p. 1223).

Few human studies have attempted to link E/I imbalance and connectivity. One such recent comprehensive study of brainwide connectivity found that hyperconnectivity, in individuals with autism, was correlated with fluctuations in response amplitude (Supekar et al., 2013), which can be a consequence of networks striving to restore E/I imbalance. Network homeostasis can also help understand apparent contradictory connectivity findings because both decreased and increased connectivity can be a response to E/I imbalance. Noise increases in an additive way with every synaptic contact the information needs to cross. Averaging of input (increased local connectivity) and decreasing the number of relay synaptic connections (decreased long-distance connectivity) both result in decreasing noise levels (Faisal, Selen, \& Wolpert, 2008). Decreased long-distance connectivity may also be adaptive because it allows for local hubs to deal with E/I imbalance independent of each other. Because subsequent stages of processing cannot extract more noise than is present at earlier stages (data-processing inequality problem), organisms pay a high metabolic and structural price at the first stage of processing in order to decrease noise (Faisal et al., 2008). It is thus possible that, developmentally, an increase in local connectivity precedes a decrease (or slower development of) long-distance connectivity.

\section{Adjusting developmental trajectories}

Neural "noise," originating either in the neurons themselves or as a result of noisy environments, can also have a bearing on the course of developmental events. Experiments on the sensitive period for establishing maps of sound frequencies (tonotopic maps) in the primary auditory cortex of rat pups have shown that while temporally modulated noise can degrade the tonotopic map and prematurely close the sensitive period (Zhang, Bao, \& Merzenich, 2002), exposure to continuous and unmodulated noise similarly disrupts the formation of the tonotopic map but actually prolongs the sensitive period (Chang \& Merzenich, 2003). Under both sets of circumstances, however, the auditory cortex comes out of the sensitive period in an undifferentiated and unstable state (Rubenstein \& Merzenich, 2003). The cases where sensitive periods are prolonged in the absence of the relevant input abound (Doupe \& Kuhl, 1999; Katz \& Shatz, 1996). Nevertheless, the above conclusion should not be thought of purely in terms of externally induced noise. If a newborn has a brain that is differentially noisy or inefficient, then his or her processing of the early environment may consequently be less consistent and reliable than is typical. The processes of adaptation of timing of brain development discussed above may then kick in, prolonging the plasticity of cortical circuits beyond their typical timetable.

Specifically, in some cases that lead to autism, the early environment may be sampled with poor fidelity, with a particular cost to the most dynamic and least easily predictable elements of the external environment. In many cases, these are associated with the social world and the complex and pseudo-predictable interactions with other humans. A mild degree of noisy sampling may lead to prolonged plasticity 
and delays in the subsequent steps of brain development that may be specific to the most complex aspects of the early environment. These risks will obviously be increased in poorquality early environments, and possibly also in individuals with genetic variants that bias them to a relatively fast or slow trajectory of brain growth. It is interesting that the timing of individual differences in plasticity is likely to be more affected by moderately uncertain environments than in highly predictable or highly unpredictable environments, because individual differences in cue sampling can have a greater impact on conclusions drawn about environmental variables (Frankenhuis \& Panchanathan, 2011). Thus, increasing the predictability of the environment may partially offset the effects of inconsistent sampling for individuals with ASD. Further, individuals with ASD may do this themselves, by orienting to more predictable events (see the next section). Moreover, behavioral interventions that increase the clarity and predictability of social interaction and learning episodes can improve developmental trajectories for children with ASD (Dawson et al., 2012).

\section{Niche construction}

Niche construction is the process by which individuals construct an environment that suits their processing style. We hypothesize that apparent differences in attentional style in some developmental disorders could reflect the adaptive strategies of the brain, given the limitations and capacities of its processing, as opposed to inferring localizable damage to attention systems in the brain. Poor-quality synaptic processing will mean sparser and less reliable sampling of information from the environment. We propose that the attentional style characteristic of autism (i.e., overly focal, biased to simple repetitive events, and highly alert) is the result of an atypical brain adapting to the pace and quantity of information flow. Poor signal to noise ratio within each sensory modality may result in poor cross-modal integration, which could lead to greater interference and thus worse performance from using one modality alone. Thus, an overly focal style of attention (characteristic of autism) could reflect parallel processing limits that mean it is beneficial to restrict sensory input to a single channel or area of external space. A second example is selecting for attention and processing elements of the environment that are more temporally predictable and that could better suit a brain with unusual signal to noise ratios. A preference for intermediary levels of predictability in sensory stimuli has been documented in infants as young as 8 months of age (Kidd et al., 2012) and is believed to reflect a bias to attend to learnable information (as compared to highly unpredictable sequences). Learning highly complex social rules (e.g., We take our shoes off when entering a house unless it's a public venue) requires frequently sampling world events to compute their probabilities and dependencies. Variability in sampling (e.g., due to poor signal to noise ratio) possibly affects the learning of higher complexity rules more than it would affect simple rules. Learnability is therefore shifted to- ward simpler structures, which often tend to be repetitive, mechanical, self-controlled stimulation, to the detriment of typical social interaction.

Because tools available for niche construction (such as motor skills, control of action, and independence) develop gradually, behavioral patterns that reflect niche construction would be expected to emerge over early development. Prospective studies of infants with later autism have revealed remarkably normal patterns of social orienting and engagement in the first months of life (for review, see Jones, Gliga, Bedford, Charman, \& Johnson, 2013). However, the infancy period is characterized by a gradual withdrawal from social interaction, such that by age 2 to 3 years, toddlers with ASD are significantly less likely to seek social contact (Jones \& Klin, 2013; Ozonoff et al., 2010). Seeking refuge in the more predictable nonsocial world and withdrawing from the less predictable and multisensory social world may thus represent an adaptive response to difficulties in processing more complex inputs.

\section{Predictions}

The hypothesis presented here goes beyond the idea that autism can be caused by increased levels of noise due to an increased ratio of neural excitation to inhibition (Rubenstein \& Merzenich, 2003) in several ways: (a) it also remains possible that reductions in background neural noise, or other changes in signal to noise ratio, could have similar computational consequences; (b) disturbances of excitation/inhibition, or other changes in synaptic efficiency, need only be transient in development to change the subsequent developmental trajectory; (c) the disturbance could be mild and widespread, and not localized to particular circuits or regions, but still differentially affect domains of processing that require rapid temporal prediction, or that are dynamic and uncertain as opposed to repetitive and high contrast; and (d) the resulting developmental trajectory will reflect the multiple processes of ontogentic adaptation discussed earlier and result in a variant of the typical trajectory. The perspective on the final common pathway for autism outlined above yields a number of predictions that can be assessed against currently available data and suggests new lines of work that are hitherto unexplored.

Measures of altered signal processing. A number of recent reports suggest increased intertrial variability in response to sensory stimulation in adults diagnosed with autism (e.g., Dinstein et al., 2012; Milne, 2011). While these reports appear inconsistent with the hypothesis above, it is also worth noting that decreases in the fidelity of processing could be transient, but still be sufficient to induce deviation from the typical developmental trajectory. Based on the literature on developmental timing reviewed above, we predict that longitudinal studies will reveal that the largest atypicalities in measures of neural signal processing quality will be apparent in early postnatal development. Further, because core behaviors 
associated with ASD are proposed to represent adaptive responses to early disruptions in synaptic function that may be later corrected (at least partially) by homeostatic mechanisms, neural noise in early development may be more closely correlated with later phenotypic measures than with measures of neural noise taken concurrently.

Improving signal to noise quality in adulthood will not ameliorate core symptoms of autism in some cases. One provocative implication of our proposal is that treatments targeted at the synaptic dysfunction assumed to be the original neural "cause" of autism may be ineffective at altering the core behavioral symptoms of autism in adulthood, because by that point the alternative developmental trajectory has become embedded. How can this be reconciled with reports of apparent "rescuing" of mice with mutations in ASD-related genes? Specifically, Michalon et al. (2012) found that mGluR5 inhibition in fmrl knockout mice (a model for fragile $\mathrm{X}$ syndrome) rescues learning and memory deficits, hyperreactivity to sensory stimuli, elevated locomotor activity, and a range of neural markers that include elevated sensitivity to epilepsy, excessive protein synthesis, long-term depression, activity of signaling pathways, and macro-orchidism. However, it is important to note that this study did not include a control group of the same age, because this would have told us if the phenotype was reversed to the wild type phenotype. One intriguing possibility is that the different aspects of the fmrl knockout mice phenotype that have been rescued are a reflection of an ongoing neural deficit, while other aspects (e.g., social and communication symptoms) are adaptive responses to earlier synaptic dysfunction, and therefore cannot be rescued in adulthood. In contrast, the same treatments applied during infancy would be predicted to have strong effects on core social and communication symptoms. It is interesting that, although autism is highly comorbid with low IQ and the two are inversely correlated, IQ and autism traits are substantially genetically independent (e.g., Hoekstra, Happé, BaronCohen, \& Ronald, 2010). We also need to consider that while in humans cases of de novo mutations may be more likely to lead to lifelong atypicality in synaptic function, risk factors associated with extremes of typical genetic variation (and thus, family risk) are more likely to involve transient disturbance or imbalance of synaptic function.

Delayed specialization of nexus regions. Delayed or atypical specialization of function and associated microstructure can occur as a result of a changed signal to noise ratio in cortical circuits during development (Rubenstein \& Merzenich, 2003). However, in addition to this, we predict that the delayed or atypical specialization will be specifically observed for regions associated with complex, dynamic, and less predictable stimuli (features that typically characterize the social world). A widely held view is that regions such as the fusiform face area and the temporal-parietal junction (TPJ) become recruited into the cortical social brain network because their patterns of primary inputs and outputs make them suitable as a "nexus" region for integrating across sensory channels with high temporal precision (Carter \& Huettel, 2013; Johnson, 2011b). For example, Carter and Huettel (2013) argue that the TPJ is frequently active in social cognitive tasks because it is located at the anatomical convergence of several different critical pathways of information processing, and the overlap and integration of these different streams leads to novel secondary computations, such as decision making within a social context. In this context, it is not surprising that evidence shows that the TPJ is often differentially impaired in adults diagnosed with ASD (Lombardo, Chakrabarti, Bullmore, \& Baron-Cohen, 2011). If a lack of specialization of the TPJ is an early critical event on the causal pathway resulting in ASD, this should be observed relatively early in postnatal life. A recent report from a study of infants at risk for later autism provides some evidence in support of this proposal (Lloyd-Fox et al., 2013). Lloyd-Fox et al. showed diminished hemodynamic responses in temporal lobe regions that are typically specialized for processing visual and auditory social stimuli in 4- to 6-month-old infants at family risk for later autism. Data suggested larger responses to nonsocial stimuli in the same brain region in high-risk infants, consistent with a lack of specialization of these regions of the temporal lobe.

Delayed perceptual narrowing. The proposition that sensitive/critical periods in infancy will be extended or delayed yields other predictions. For example, between 6 and 12 months, typically developing infants show a declining ability to distinguish phonemes from languages to which they are not exposed; similar effects are seen for music and face processing (for a review, see Lewkowicz \& Ghazanfar, 2009). Delayed specialization would prolong this process of perceptual narrowing, leading to the prediction that infants who go on to be diagnosed with autism will show an enhanced ability to discriminate faces, phonemes, and voices from unfamiliar categories (e.g., nonnative speakers) in the second year of life and delayed onset of other-race effects. It is interesting that delayed perceptual narrowing has recently been observed in premature infants (Jansson-Verkasalo et al., 2010), a group who have a substantially higher risk of autism (Johnson et al., 2010). Further, a key indicator of the sensitive period for ocular dominance plasticity is the window within which treatments for amblyopia are most effective. In typically developing individuals, effectiveness of treatment diminishes after the age of 7 years, although there are substantial individual differences in response to treatment after that period (Scheiman et al., 2008). Similarly, musical training leads to an expanded auditory cortical representation, but only before 9 years of age (Pantev et al., 1998). If sensitive periods are disrupted in individuals with autism, we would predict that treatment efficacy for amblyopia may have a significantly different time course. There is some evidence from animal models of autism that this may be the case (for a review, see LeBlanc \& Fagiolini, 2011). Leaving sensitive periods open for longer will also mean that correlations between brain 
measures and environmental factors will show different developmental profiles. For example, within a group of highrisk infants, Baker, Messinger, Lyons, and Grantz (2010) found that higher maternal sensitivity at 18 months predicted language growth between 2 and 3 years of age for children with emergent ASD only. Possibly, this relationship would have been present earlier in typically developing children. Hypothesis-driven tests of such relations will be an important target for future work with high-risk infant populations.

Heritability of adaptive mechanisms and heritability of synaptic atypicality are separable. Variation in adaptive mechanisms is also heritable (Frankenhuis \& Panchanathan, 2011). For example, the literature on differential susceptibility and biological susceptibility to context (e.g., Belsky \& Pluess, 2009; Boyce \& Ellis, 2005) has examined the role of genetic polymorphisms in response to environmental adversity or advantage, revealing a number of genes that appear to influence the degree to which an individual adapts to his or her early environmental experiences. "Orchid" children, or those who adapt readily, tend to show more differential outcomes in favorable versus adverse environments than do "dandelion" children, who appear to be less influenced by external conditions (Ellis \& Boyce, 2008). For example, children with the 7-repeat allele of the DRD4 dopamine gene are the least social in the absence of positive parenting, but they are the most social in the presence of positive parenting (Knafo et al., 2011). Such differential susceptibility factors possibly represent variation in adaptive tuning to early environmental variables; it has been suggested that DRD4-7 represents a "plasticity" gene (Pluess \& Belsky, 2009).

Plasticity genes are expected to also affect the development of individuals growing up in a typical environment but whose processing of the environment is atypical, as is the case of children with ASD. Having the seven-repeat allele of the DRD4 dopamine gene might lead, in these cases, to greater behavioral symptoms of autism (adaptations). For example, Reiersen and Todorov (2011) found that symptoms of autism within individuals with ADHD are higher in those with the seven-repeat DRD4 allele. Testing this hypothesis within other populations with genetic risk factors for autism is an important step for future work.

It is likely that each of the adaptive mechanisms we described rely on different genetic factors. Thus, although all the adaptive changes are expected to take place as a result of a common trigger (i.e., the synaptic malfunction), individual variability may result from allelic variation within genes involved in neuronal homeostasis, sensory period timing, or niche construction.

\section{Limitations and Future Directions}

Although we built the current proposal on the assumption that ASD results from nonoptimal synaptic function, but intact adaptive mechanisms (which leads to behavioral adaptations that constitute part of the ASD symptomatology), it remains possible that some degree of phenotypic variability within individuals with ASD relates to the degree to which adaptation mechanisms themselves are compromised by synaptic malfunction. Other neurodevelopmental disorders appear to be linked to deficits in the process of homeostasis itself. For example, lack or loss of function mutations in the Rett gene methyl $\mathrm{CpG}$ binding protein $2(\mathrm{MeCP} 2)$ disrupt homeostatic network plasticity in the developing cortex (Blackman, Djukic, Nelson, \& Turrigiano, 2012). Further, lack of fragile $\mathrm{X}$ mental retardation protein (fMRP) disrupts a particular type of homeostatic plasticity in developing hippocampal networks (Soden \& Chen, 2010) and may also explain attempted but failed homeostatic responses to disrupted synaptic functioning in the amygdala (Vislay et al., 2013). Disruptions in inhibitory/excitatory balance likely extend to networks like the PFC that are involved in adaptive processes (e.g., Zikopoulos \& Barbas, 2013), Thus, considering the degree to which adaptive processes are themselves compromised may be key to understanding variability within groups of individuals with ASD.

A further step will be to examine the relation between developmental regression (observed in at least $30 \%$ of individuals; Ozonoff et al., 2011) and the operation of core adaptive mechanisms. Regressive events may reflect failed homeostatic mechanisms. When a local minimum cannot be reached through cellular or network homeostatic reorganization, the network may reach a point of catastrophic collapse. Some adults with deletions that include the SH3 and multiple ankyrin repeat domains 3 (SHANK3) protein (associated with significant deficits in excitatory synaptic transmission; Shcheglovitov et al., 2013) experience sudden-onset regression in adult life after a significant life event such as a seizure, septic shock, or malignant neuroleptic syndrome (Denayer et al., 2012). More gradual regression during development was observed in all these individuals. Investigations of patients with Phelan-McDermid syndrome (typically associated with a $22 q 13$ deletion spanning the SHANK3 region) may provide insight into the mechanisms underlying this regression.

Despite these limitations, we believe that the views outlined in this paper have significant implications for both the development study of autism in the early years and the conclusions that can be drawn from adult studies of those with developmental disorders.

1. Studies of the neural basis of the key symptoms of autism in adults are essentially investigations of the brain's ability to adapt and reorganize in the face of diffuse and widespread synaptic differences (although these original problems may persist into adulthood in some cases). These synaptic differences occur at an earlier point in development and affect the fidelity of information processing. Similar to the study of recovery of function following focal perinatal brain damage, by adulthood it is the degree and nature of brain reorganization that can be studied, but we cannot learn more about the original cause without recourse to 
studying the age of the original injury. Thus, to the extent our views are correct, one cannot directly study in adults the original causes of autism (at least in mild and moderate cases). Ironically, there are few neuroimaging studies of the severe cases of adult autism where the original synaptic causes are most likely to still be present. We note that understanding the causes of autism is going to be more challenging than that of perinatal brain damage in at least three ways: (a) we don't know precisely when in development the critical deviation events occur; (b) the neural factors are diffuse and widespread in autism and likely to affect some types of information processing more than others; and (c) we do not know with certainty which parts of the brain are unaffected, and are thus able to efficiently compensate.

2. Altering signal to noise ratios in neural processing can be the result of a variety of different molecular and genetic factors, including the balance between excitatory and inhibitory neurotransmitters. Thus, according to our view, there will be multiple, different molecular and genetic pathways capable of leading to autism, as long as they have the net effect of disturbing the typical balance that leads high-fidelity synaptic processing. Environmental instability or sensory disturbances could potentially have similar, or compounding, effects. To make the situation even more complex for genetic and molecular factors, by midchildhood a variety of different compensatory and adaptive process will have been at work, each with its own genetic correlates. In other words, the reason why the genetics of autism has proved so challenging is because a variety of different early challenges all result in the same adaptive response from the brain. Autism as a diagnostic category is best considered as the end result of an alternative developmental trajectory, a common adaptive response to a variety of different early brain function problems.

3. A major issue in developmental disorders is that of selectivity. Which factors determine whether a child develops the symptoms of autism as opposed to ADHD, or receives both diagnoses? Two alternatives are evident from our analyses. More traditionally, the neural or transmitter systems affected differ for the two diagnoses. However, given the large degree of genetic overlap between these diagnoses a second possibility is that it is the developmental stage at which the diffuse and widespread neural detuning occurs that determines the nature of the adaptive responses, and therefore the behavioral traits that emerge. In this respect, it is perhaps worthy of note that the behavioral traits that lead to a diagnosis of ADHD typically emerge several years after the appearance of those associated with autism. Possibly the phase of postnatal development affected determines the nature of the ontogenetic adaptive response, and thus the clinical profile that results. Thus, we suggest there may be a family of such adaptation syndromes. One prediction here is that genes associated with ADHD will have expression patterns that peak later in development than those associated with ASD; systematic comparisons of this nature are an important target for future work on the human epigenome.

4. One important concept to be tackled in future work is how to measure the degree of adaptation of particular individuals. Traditional views of resilience and adaptation have taken "typicality" as the desired end state. Thus, resilience would be expected to be reflected in better social and communication skills, lower rates of depression, and so on. However, the core proposal here is that adaptive processes are designed to ensure an optimal outcome for the individual brain, given its processing style and environmental inputs. Some of these adaptations may act to increase apparent "typicality," such as in the case of the use of alternate brain mechanisms to produce the same surface behavior, or the PFC-mediated use of alternate strategies to achieve the same goal. However, others may result in what appears to be greater atypicality, such as niche construction favoring social withdrawal.

Another important possibility is that if early synaptic dysfunction is transient, adaptation processes may be triggered that optimize the brain to a processing style that characterized its interactions with the environment during an earlier stage of development. Because plasticity is greater in early development, adaptation after synaptic dysfunction has disappeared may be more limited and may result in a mismatch between phenotype and the experienced environment. These arguments are similar to those made for prenatal exposure to malnutrition, which is thought to trigger the development of metabolic processes designed to retain and store insulin and fatty acids (Barker \& Clark, 1997). When the individual encounters plentiful food later in life, individuals are at increased risk for health problems that are not seen if the postnatal environment continues to lack resources (Stanner \& Yudkin, 2001).

These questions become most pressing when considering early intervention. To an extent, the viewpoint espoused in this paper strongly supports the value of early (prodromal) intervention. The adaptive response of prolonging neural plasticity may actually increase the temporal window within which early intervention could be effective. It is possible that, in at least some cases of children at risk, we could prevent or alleviate the emergence of the full symptoms of the condition by treating the original synaptic dysfunction in very early development. However, treatments focused on boosting adaptive mechanisms may have more mixed results. Improving mechanisms that are likely to increase the use of alternate brain networks to attain key goals (such as executive functioning systems) is likely to improve quality of life for affected individuals. A number of groups have recently developed EF skills training programs suitable for young children (Wass, Scerif, \& Johnson, 2012), and an exciting direction for future research will be to see whether such training regimes applied to populations of infants at risk will improve outcome in some individuals. However, enhancing other adaptive 
mechanisms in early development may be counterproductive in the case of individuals with a transient synaptic problem. Here, increasing the likelihood that individuals will adapt to a transient atypicality in processing style may serve to increase later symptoms of autism. For individuals with a more persistent synaptic problem, adaptation may be essential to later quality of life; later interventions that attempt to reduce behavioral symptoms of autism may be inadvertently removing a core adaptation to synaptic dysfunction. Navigating these difficult issues will be crucial as groups begin to design interventions targeted at infants at risk for autism.

In conclusion, we have reviewed evidence supporting the view that a range of genetic and molecular factors can cause widespread mild synaptic dysregulation during early postnatal development. This, in turn, elicits a series of compensatory and adaptive processes that trigger an alternative trajectory of

\section{References}

Alvarez, J. A., \& Emory, E. (2006). Executive function and the frontal lobes: A meta-analytic review. Neuropsychology Review, 16, 17-42.

Baker, J. K., Messinger, D. S., Lyons, K. K., \& Grantz, C. J. (2010). A pilot study of maternal sensitivity in the context of emergent autism. Journal of Autism and Developmental Disorders, 40, 988-999.

Barker, D. J., \& Clark, P. M. (1997). Fetal undernutrition and disease in later life. Reviews of Reproduction, 2, 105-112.

Barker, D. J. P. (1994). The fetal origins of adult disease Proceedings of the Royal Society of London B, 262, 37-43.

Belsky, J., \& Pluess, M. (2009). Beyond diathesis stress: Differential susceptibility to environmental influences. Psychological Bulletin, 135, 885908.

Ben-Ari, Y. (2002). Excitatory actions of GABA during development: The nature of the nurture. Nature Reviews Neuroscience, 3, 728-739.

Ben-Ari, Y., Khalilov, I., Kahle, K. T., \& Cherubini, E. (2012). The GABA excitatory/inhibitory shift in brain maturation and neurological disorders. Neuroscientist, 18, 467-486.

Blackman, M. P., Djukic, B., Nelson, S. B., \& Turrigiano, G. G. (2012). A critical and cell-autonomous role for $\mathrm{MeCP} 2$ in synaptic scaling up. Journal of Neuroscience, 32, 13529-13536.

Boyce, W. T., \& Ellis, B. J. (2005). Biological sensitivity to context: I. An evolutionary-developmental theory of the origins and functions of stress reactivity. Development and Psychopathology, 17, 271-301.

Bullmore, E., \& Sporns, O. (2012). The economy of brain network organization. Nature Reviews Neuroscience, 13, 336-349.

Cahalane, D. J., Clancy, B., Kingsbury, M. A., Graf, E., Sporns, O., \& Finlay, B. L. (2011). Network structure implied by initial axon outgrowth in rodent cortex: Empirical measurement and models. PLOS ONE, 6, e16113.

Carter, R. M., \& Huettel, S. A. (2013). A nexus model of the temporal-parietal junction. Trends in Cognitive Sciences, 17, 328-336.

Castellanos, F. X., \& Proal, E. (2012). Large-scale brain systems in ADHD: Beyond the prefrontal-striatal model. Trends in Cognitive Sciences, 16, 17-26.

Chang, E. F., \& Merzenich, M. M. (2003). Environmental noise retards auditory cortical development. Science, 300, 498-502.

Chiao, J. Y., \& Ambady, N. (2010). Cultural neuroscience. In S. Kitayama \& D. Cohen (Eds.), Handbook of cultural psychology. New York: Guilford Press.

Chih, B., Engelman, H., \& Scheiffele, P. (2005). Control of excitatory and inhibitory synapse formation by leuroligins. Science, 307, 1324-1328.

Cicchetti, D. (2013). Resilient functioning in maltreated children: Past, present, and future perspectives. Journal of Child Psychology and Psychiatry, $54,402-422$.

Cicchetti, D., \& Curtis, W. J. (2007). Multilevel perspectives on pathways to resilient functioning. Development and Psychopathology, 19, 627-629.

Clancy, B., Darlington, R. B., \& Finlay, B. L. (2000). The course of human events: Predicting the timing of primate neural development. Developmental Science, 3, 57-66. subsequent development. We propose that it is these speciestypical adaptive processes that underlie the majority of the phenotype traditionally associated with autism. Specifically, changes in connectivity and different patterns of activation revealed by functional neuroimaging may result from reorganization and redundancy; developmental delays may reflect a delayed timetable of specialization that allows a broader time window for environmental sampling; and restricted interests and social withdrawal may represent construction of an ecological niche that best suits a brain with "noisy" signal processing. Understanding the nature of autism requires us to study the intricate interaction between atypical early processing and species-typical adaptation responses over development. We speculate that autism may be only one of several developmental disorders defined by a phenotype that results from processes of adaptation.

Cobos, I., Calcagnotto, M. E., Vilaythong, A. J., Thwin, M. T., Noebels, J. L., Baraban, S. C., et al. (2005). Mice lacking Dlx1 show subtype-specific loss of interneurons, reduced inhibition and epilepsy. Nature $\mathrm{Neu}-$ roscience, 8, 1059-1068.

Cohen Kadosh, K., Henson, R. N., Cohen Kadosh, R., Johnson, M., \& Dick, F. (2010). Task-dependent activation of face-sensitive cortex: An fMRI adaptation study. Journal of Cognitive Neuroscience, 22, 903-917.

Courchesne, E., Pierce, K., Schumann, C. M., Redcay, E., Buckwalter, J. A., Kennedy, D. P., et al. (2007). Mapping early brain development in autism. Neuron, 56, 399-413.

Curtis, W. J., \& Cicchetti, D. (2003). Moving research on resilience into the 21st century: Theoretical and methodological considerations in examining the biological contributors to resilience. Development and Psychopathology, 15, 773-810.

Davis, G., \& Plaisted-Grant, K. (in press). Low endogenous neural noise in autism. Autism. doi:10.1177/1362361314552198

Dawson, G., Jones, E. J. H., Merkle, K., Venema, K., Lowy, R., Faja, S., et al (2012). Early behavioral intervention is associated with normalized brain activity in young children with autism. Journal of the American Academy of Child \& Adolescent Psychiatry, 51, 1150-1159.

Dehaene-Lambertz, G., Hertz-Pannier, L., \& Dubois, J. (2006). Nature and nurture in language acquisition: Anatomical and functional brain-imaging studies in infants. Trends in Neurosciences, 29, 367-373.

Denayer, A., Van Esch, H., de Ravel, T., Frijns, J.-P., Van Buggenhout, G., Vogels, A., et al. (2012). Neuropsychopathology in 7 patients with the 22 q13 deletion syndrome: Presence of bipolar disorder and progressive loss of skills. Molecular Syndromology, 3, 14-20.

Desai, N. S., Cudmore, R. H., Nelson, S. B., \& Turrigiano, G. G. (2002). Critical periods for experience-dependent synaptic scaling in visual cortex. Nature Neuroscience, 5, 783-789.

Dinstein, I., Heeger, D. J., Lorenzi, L., Minshew, N. J., Malach, R., \& Behrmann, M. (2012). Unreliable evoked responses in autism. Neuron, 75, 981-991.

Dinstein, I., Pierce, K., Eyler, L., Solso, S., Malach, R., Behrmann, M., et al. (2011). Disrupted neural synchronization in toddlers with autism. Neuron, 70, 1218-1225.

Doria, V., Beckmann, C. F., Arichi, T., Merchant, N., Groppo, M., Turkheimer, F. E., et al. (2010). Emergence of resting state networks in the preterm human brain. Proceedings of the National Academy of Sciences, 107, 20015-20020.

Doupe, A. J., \& Kuhl, P. K. (1999). Birdsong and human speech: Common themes and mechanisms. Annual Review of Neuroscience, 22, 567-631.

Ebert, D. H., \& Greenberg, M. E. (2013). Activity-dependent neuronal signalling and autism spectrum disorder. Nature, 493, 327-337.

Eichler, S. A., \& Meier, J. C. (2008). EI balance and human diseases-From molecules to networking. Frontiers in Molecular Neuroscience, 1, 2.

Ellis, B. J., \& Boyce, W. T. (2008). Biological sensitivity to context. Current Directions in Psychological Science, 17, 183-187. 
Elman, J. L. (Ed.). (1998). Rethinking innateness: A connectionist perspective on development (Vol. 10). Cambridge, MA: MIT Press.

Faisal, A. A., Selen, L. P. J., \& Wolpert, D. M. (2008). Noise in the nervous system. Nature Reviews Neuroscience, 9, 292-303.

Fassbender, C., \& Schweitzer, J. B. (2006). Is there evidence for neural compensation in attention-deficit/hyperactivity disorder? A review of the functional neuroimaging literature. Clinical Psychology Review, 26, 445-465.

Finlay, B. L., \& Darlington, R. B. (1995). Linked regularities in the development and evolution of mammalian brains. Science, 268, 1578-1584.

Finlay, B. L, Yost, J. C., \& Cheung, D. T. (2006). Developmental disorders and evolutionary expectations: Mechanisms of resilience. In M. M. Miller (Ed.), Brain development: Normal processes and the effects of alcohol and nicotine (pp. 105-120). Oxford: Oxford University Press.

Flynn, E. G., Laland, K. N., Kendal, R. L., \& Kendal, J. R. (2013). Target Article with Commentaries: Developmental niche construction. Developmental Science, 16, 296-313.

Frankenhuis, W. E., \& Panchanathan, K. (2011). Balancing sampling and specialization: An adaptationist model of incremental development. Proceedings of the Royal Society B: Biological Sciences, 278, 35583565.

Frankenhuis, W. E., Panchanathan, K., \& Clark Barrett, H. (2013). Bridging developmental systems theory and evolutionary psychology using dynamic optimization. Developmental Science, 16, 584-598.

Fransson, P., Åden, U., Blennow, M., \& Lagercrantz, H. (2011). The functional architecture of the infant brain as revealed by resting-state fMRI. Cerebral Cortex, 21, 145-154.

Friston, K. J., \& Price, C. J. (2001). Dynamic representations and generative models of brain function. Brain Research Bulletin, 54, 275-285.

Gavin-Smyth, J., Wang, W., Butler, I., \& Ferguson, E. L. (2013). A genetic network conferring canalization to a bistable patterning system in drosophila. Current Biology, 23, 2296-2302.

Geschwind, D. H., \& Levitt, P. (2007). Autism spectrum disorders: Developmental disconnection syndromes. Current Opinion in Neurobiology, 17, $103-111$.

Gilbert, S. L., Dobyns, W. B., \& Lahn, B. T. (2005). Genetic links between brain development and brain evolution. Nature Reviews Genetics, 6, 581590.

Goel, A., \& Lee, H.-K. (2007). Persistence of experience-induced homeostatic synaptic plasticity through adulthood in superficial layers of mouse visual cortex. Journal of Neuroscience, 27, 6692-6700.

Gogtay, N., Giedd, J. N., Lusk, L., Hayashi, K. M., Greenstein, D., Vaituzis, A. C., et al. (2004). Dynamic mapping of human cortical development during childhood through early adulthood. Proceedings of the National Academy of Sciences, 101, 8174-8179.

Gottlieb, J., Oudeyer, P., Lopes, M., \& Baranes, A. (2013). Information-seeking, curiosity, and attention: Computational and neural mechanisms. Trends in Cognitive Sciences, 17, 585-593.

Graf, E. R., Zhang, X., Jin, S.-X., Linhoff, M. W., \& Craig, A. M. (2004). Neurexins induce differentiation of GABA and glutamate postsynaptic specializations via neuroligins. Cell, 119, 1013-1026.

Grossmann, T. (2013). Mapping prefrontal cortex functions in human infancy. Infancy, 8, 303-324.

Halperin, J. M., \& Schulz, K. P. (2006). Revisiting the role of the prefrontal cortex in the pathophysiology of attention-deficit/hyperactivity disorder. Psychological Bulletin, 132, 560-581.

Harlow, E. G., Till, S. M., Russell, T. A., Wijetunge, L. S., Kind, P., \& Contractor, A. (2010). Critical period plasticity is disrupted in the barrel cortex of FMR1 knockout mice. Neuron, 65, 385-398.

Haxby, J. V., Hoffman, E. A., \& Ida Gobbini, M. (2000). The distributed human neural system for face perception. Trends in Cognitive Sciences, 4, 223-233.

Hensch, T. K. (2005). Critical period plasticity in local cortical circuits. Nature Reviews Neuroscience, 6, 877-888.

Hensch, T. K., Fagiolini, M., Mataga, N., Stryker, M. P., Baekkeskov, S., \& Kash, S. F. (1998). Local GABA circuit control of experience-dependent plasticity in developing visual cortex. Science, 282, 1504-1508.

Hoekstra, R. A., Happé, F., Baron-Cohen, S., \& Ronald, A. (2010). Limited genetic covariance between autistic traits and intelligence: Findings from a longitudinal twin study. American Journal of Medical Genetics, 153B, 994-1007.

Hoerder-Suabedissen, A., Oeschger, F. M., Krishnan, M. L., Belgard, T. G., Wang, W. Z., Lee, S., et al. (2013). Expression profiling of mouse subplate reveals a dynamic gene network and disease association with autism and schizophrenia. Proceedings of the National Academy of Sciences, $110,3555-3560$.

Howard, M. A., Rubenstein, J. L. R., \& Baraban, S. C. (2013). Bidirectional homeostatic plasticity induced by interneuron cell death and transplantation in vivo. Proceedings of the National Academy of Sciences, 111, 492-497.

Jansson-Verkasalo, E., Ruusuvirta, T., Huotilainen, M., Alku, P., Kushnerenko, E., Suominen, K., et al. (2010). Atypical perceptual narrowing in prematurely born infants is associated with compromised language acquisition at 2 years of age. BMC Neuroscience, 11,88 .

Johnson, M. H.. (1990). Cortical maturation and the development of visual attention in early infancy. Journal of Cognitive Neuroscience, 2, 81-95.

Johnson, M. H. (2001). Functional brain development in humans. Nature Reviews Neuroscience, 2, 475-483.

Johnson, M. H. (2005). Subcortical face processing. Nature Reviews Neuroscience, 6, 766-774.

Johnson, M. H., Griffin, R., Csibra, G., Halit, H., Farroni, T., De Haan, M., et al. (2005). The emergence of the social brain network: Evidence from typical and atypical development. Development and Psychopathology, $17,599-619$.

Johnson, M. H. (2011a). Interactive specialization: A domain-general framework for human functional brain development. Developmental Cognitive Neuroscience, 1, 7-21.

Johnson, M. H. (2011b). Face processing as brain adaptation at multiple time scales. Quarterly Journal of Experimental Psychology, 64, 1873-1888.

Johnson, M. H. (2012). Executive function and developmental disorders: The flip side of the coin. Trends in Cognitive Sciences, 16, 454-457.

Johnson, M. H. (in press). Neurobiological perspectives on developmental psychopathology. In M. Rutter et al. (Eds.), Rutter's child and adolescent psychology (6th ed.). Hoboken, NJ: Wiley.

Johnson, S., Hollis, C., Kochhar, P., Hennessy, E., Wolke, D., \& Marlow, N. (2010). Psychiatric disorders in extremely preterm children: Longitudinal finding at age 11 years in the EPICure study. Journal of the American Academy of Child \& Adolescent Psychiatry, 49, 453-463.

Jones, E. J. H., Gliga, T., Bedford, R., Charman, T., \& Johnson, M. H. (2013). Developmental pathways to autism: A review of prospective studies of infants at risk. Neuroscience \& Biobehavioral Reviews. Advance online publication. doi:10.1016/j.neubiorev.2013.12.001

Jones, W., \& Klin, A. (2013). Attention to eyes is present but in decline in $2-$ to 6-month-old infants later diagnosed with autism. Nature. Advance online publication. doi:10.1038/nature 12715

Kaiser, M. D., Hudac, C. M., Shultz, S., Lee, S. M., Cheung, C., Berken, A. M., et al. (2010). Neural signatures of autism. Proceedings of the National Academy of Sciences, 107, 21223-21228.

Kang, H. J., Kawasawa, Y. I., Cheng, F., Zhu, Y., Xu, X., Li, M., et al. (2011) Spatio-temporal transcriptome of the human brain Nature, 478, 483-489.

Karatoreos, I. N., \& McEwen, B. S. (2013). Annual Research Review: The neurobiology and physiology of resilience and adaptation across the life course. Journal of Child Psychology and Psychiatry, 54, 337-347.

Karmiloff-Smith, A., Casey, B. J., Massand, E., Tomalksi, P., \& Thomas, M. S. C. (2014). Environmental and genetic influences on neurocognitive development. Clinical Psychological Science. Advance online publication.

Karmiloff-Smith, A., Thomas, M., Annaz, D., Humphreys, K., Ewing, S., Brace, N., et al. (2004). Exploring the Williams syndrome face-processing debate: The importance of building developmental trajectories. Journal of Child Psychology and Psychiatry, 45, 1258-1274.

Katz, L. C., \& Shatz, C. J. (1996). Synaptic activity and the construction of cortical circuits. Science, 274, 1133-1138.

Kidd, C., Piantadosi, S. T., \& Aslin, R. N. (2012). The Goldilocks effect: Human infants allocate attention to visual sequences that are neither too simple nor too complex. PLOS ONE, 7, e36399.

Knafo, A., Israel, S., \& Ebstein, R. P. (2011). Heritability of children's prosocial behavior and differential susceptibility to parenting by variation in the dopamine receptor D4 gene. Development and Psychopathology, 23 , 53-67.

Kroon, T., Sierksma, M. C., \& Meredith, R. M. (2013). Investigating mechanisms underlying neurodevelopmental phenotypes of autistic and intellectual disability disorders: A perspective. Frontiers in Systems Neuroscience, 7,75 .

Laland, K. N., Odling-Smee, J., \& Feldman, M. W. (2000). Niche construction, biological evolution, and cultural change. Behavioral and Brain Sciences, 23, 131-146.

LeBlanc, J. J., \& Fagiolini, M. (2011). Autism: A “critical period" disorder? Neural Plasticity, 2011. 
Lewkowicz, D. J., \& Ghazanfar, A. A. (2009). The emergence of multisensory systems through perceptual narrowing. Trends in Cognitive Sciences, 13, 470-478.

Li, Y., Yang, J., Scherf, K. S., \& Li, P. (2013). Two faces, two languages: An fMRI study of bilingual picture naming. Brain and Language, 127, 452462.

Liston, C., Malter Cohen, M., Teslovich, T., Levenson, D., \& Casey, B. J. (2011). Atypical prefrontal connectivity in attention-deficit/hyperactivity disorder: Pathway to disease or pathological end point? Biological Psychiatry, 69, 1168-1177.

Lloyd-Fox, S., Blasi, A., Elwell, C. E., Charman, T., Murphy, D., \& Johnson, M. H. (2013). Reduced neural sensitivity to social stimuli in infants at risk for autism. Proceedings of the Royal Society B: Biological Sciences, 280, 20123026.

Lombardo, M. V., Chakrabarti, B., Bullmore, E. T., \& Baron-Cohen, S. (2011). Specialization of right temporo-parietal junction for mentalizing and its relation to social impairments in autism. Neurolmage, 56, 18321838.

Losh, M., Adolphs, R., Poe, M. D., Couture, S., Penn, D., Baranek, G. T., et al. (2009). Neuropsychological profile of autism and the broad autism phenotype. Archives of General Psychiatry, 66, 518-526.

Luthar, S. S., \& Cicchetti, D. (2000). The construct of resilience: Implications for interventions and social policies. Development and Psychopathology, $12,857-885$.

Maffei, A., Nataraj, K., Nelson, S. B., \& Turrigiano, G. G. (2006). Potentiation of cortical inhibition by visual deprivation. Nature, 443, $81-84$.

Maffei, A., Nelson, S. B., \& Turrigiano, G. G. (2004). Selective reconfiguration of layer 4 visual cortical circuitry by visual deprivation. Nature Neuroscience, $7,1353-1359$.

Maffei, A., \& Turrigiano, G. G. (2008). Multiple modes of network homeostasis in visual cortical layer 2/3. Journal of Neuroscience, 28 , 4377-4384.

Masten, A. S. (2007). Resilience in developing systems: Progress and promise as the fourth wave rises. Development and Psychopathology, 19, 921930.

McClelland, J. L., McNaughton, B. L., \& O’Reilly, R. C. (1995). Why there are complementary learning systems in the hippocampus and neocortex: Insights from the successes and failures of connectionist models of learning and memory. Psychological Review, 102, 419-457.

Menon, V. (2013). Developmental pathways to functional brain networks: Emerging principles. Trends in Cognitive Sciences, 17, 627-640.

Meredith, R. M., Dawitz, J., \& Kramvis, I. (2012). Sensitive time-windows for susceptibility in neurodevelopmental disorders. Trends in Neurosciences, 35, 335-344.

Michalon, A., Sidorov, M., Ballard, T. M., Ozmen, L., Spooren, W., Wettstein, J. G., et al. (2012). Chronic pharmacological mGlu5 inhibition corrects fragile $\mathrm{X}$ in adult mice. Neuron, 74, 49-56.

Milne, E. (2011). Increased intra-participant variability in children with autistic spectrum disorders: Evidence from single-trial analysis of evoked EEG. Frontiers in Perception Science, 2, 51.

Ozonoff, S., Iosif, A.-M., Baguio, F., Cook, I. C., Hill, M. M., Hutman, T., et al. (2010). A Prospective study of the emergence of early behavioral signs of autism. Journal of the American Academy of Child \& Adolescent Psychiatry, 49, 256-266.

Ozonoff, S., Iosif, A.-M., Young, G. S., Hepburn, S., Thompson, M., Colombi, C., et al. (2011). Onset patterns in autism: Correspondence between home video and parent report. Journal of the American Academy of Child \& Adolescent Psychiatry, 50, 796-806.

Pantev, C., Oostenveld, R., Engelien, A., Ross, B., Roberts, L. E., \& Hoke, M. (1998). Increased auditory cortical representation in musicians. $\mathrm{Na}$ ture, 392, 811-814.

Parikshak, N. N., Luo, R., Zhang, A., Won, H., Lowe, J. K., Chandran, V., et al. (2013). Integrative functional genomic analyses implicate specific molecular pathways and circuits in autism. Cell, 155, 1008-1021.

Penzes, P., Cahill, M. E., Jones, K. A., VanLeeuwen, J. E., \& Woolfrey, K. M. (2011). Dendritic spine pathology in neuropsychiatric disorders. $\mathrm{Na}$ ture Neuroscience, 14, 285-293.

Pierce, K., Müller, R.-A., Ambrose, J., Allen, G., \& Courchesne, E. (2001). Face processing occurs outside the fusiform "face area" in autism: Evidence from functional MRI. Brain, 124, 2059-2073.

Pluess, M., \& Belsky, J. (2009). Differential susceptibility to rearing experience: The case of childcare. Journal of Child Psychology and Psychiatry and Allied Disciplines, 50, 396-404.
Reiersen, A. M., \& Todorov, A. A. (2011). Association between DRD4 genotype and autistic symptoms in DSM-IV ADHD. Journal of the Canadian Academy of Child and Adolescent Psychiatry, $20,15-21$.

Rovee-Collier, C., \& Cuevas, K. (2009). Multiple memory systems are unnecessary to account for infant memory development: An ecological model. Developmental Psychology, 45, 160-174.

Rubenstein, J. L. R., \& Merzenich, M. M. (2003). Model of autism: Increased ratio of excitation/inhibition in key neural systems. Genes, Brain, and Behavior, 2, 255-267.

Scarr, S. (1992). Developmental theories for the 1990s: Development and individual differences. Child Development, 63, 1-19.

Scheiman, M. M., Hertle, R. W., Kraker, R. T., Beck, R. W., Birch, E. E., Felius, J., et al. (2008). Patching vs atropine to treat amblyopia in children aged 7 to 12 years: A randomized trial. Archives of Ophthalmology, 126, $1634-1642$.

Schiller, P. H. (1985). A model for the generation of visually guided saccadic eye movements. In D. Rose \& V. G. Dobson (Eds.), Models of the visual cortex (pp. 62-70). New York: Wiley.

Schroer, R. J., Phelan, M. C., Michaelis, R. C., Crawford, E. C., Skinner, S. A., Cuccaro, M., et al. (1998). Autism and maternally derived aberrations of chromosome 15q. American Journal of Medical Genetics, 76, 327-336.

Seybold, B. A., Stanco, A., Cho, K. K. A., Potter, G. B., Kim, C., Sohal, V. S., et al. (2012). Chronic reduction in inhibition reduces receptive field size in mouse auditory cortex. Proceedings of the National Academy of Sciences, 109, 13829-13834.

Shcheglovitov, A., Shcheglovitova, O., Yazawa, M., Portmann, T., Shu, R., Sebastiano, V., et al. (2013). SHANK3 and IGF1 restore synaptic deficits in neurons from 22q13 deletion syndrome patients. Nature, 503, 267-271.

Shen, M. D., Nordahl, C. W., Young, G. S., Wootton-Gorges, S. L., Lee, A., Liston, S. E., et al. (2013). Early brain enlargement and elevated extraaxial fluid in infants who develop autism spectrum disorder. Brain, 136, 2825-2835.

Shultz, T. R., Rivest, F., Egri, L., Thivierge, J.-P., \& Dandurand, F. (2007) Could knowledge-based neural learning be useful in developmental robotics? The case of KBCC. International Journal of Humanoid Robotics, 4, 245-279.

Soden, M. E., \& Chen, L. (2010). Fragile-X protein FMRP is required for homeostatic plasticity and regulation of synaptic strength by retinoic acid. Journal of Neuroscience, 30, 16910-16921.

Stanner, S. A., \& Yudkin, J. S. (2001). Fetal programming and the Leningrad Siege study. Twin Research, 4, 287-292.

Supekar, K., Uddin, L. Q., Khouzam, A., Phillips, J., Gaillard, W. D., Kenworthy, L. E., et al. (2013). Brain hyperconnectivity in children with autism and its links to social deficits. Cell Reports, 5, 738-747.

Sur, M., \& Leamey, C. A. (2001). Development and plasticity of cortical areas and networks. Nature Reviews Neuroscience, 2, 251-262.

Thatcher, R. W. (1992). Cyclic cortical reorganization during early childhood. Brain and Cognition, 20, 24-50.

Thivierge, J. P., Titone, D., \& Shultz, T. R. (2005). Simulating frontotemporal pathways involved in lexical ambiguity resolution. Poster presented at the 27th annual conference of the Cognitive Science Society, Stresa, Italy, July $21-23$.

Thomas, M. S. C., Knowland, V. C. P., \& Karmiloff-Smith, A. (2011). Mechanisms of developmental regression in autism and the broader phenotype: A neural network modeling approach. Psychological Review, $118,637-654$.

Toyoizumi, T., Miyamoto, H., Yazaki-Sugiyama, Y., Atapour, N., Hensch, T. K., \& Miller, K. D. (2013). A theory of the transition to critical period plasticity: Inhibition selectively suppresses spontaneous activity. Neuron, 80, 51-63.

Turrigiano, G. (2011). Too many cooks? Intrinsic and synaptic homeostatic mechanisms in cortical circuit refinement. Annual Review of Neuroscience, 34, 89-103.

Turrigiano, G. G., \& Nelson, S. B. (2004). Homeostatic plasticity in the developing nervous system. Nature Reviews Neuroscience, 5, 97-107.

Uddin, L. Q., Supekar, K., \& Menon, V. (2013). Reconceptualizing functional brain connectivity in autism from a developmental perspective. Frontiers in Human Neuroscience, 7, 458.

van den Heuvel, M. P., Stam, C. S., Kahn, R. S., \& Pol, H. E. (2009). Efficiency of functional brain networks and intellectual performance. Journal of Neuroscience, 29, 7619-7624. 
Vislay, R. L., Martin, B. S., Olmos-Serrano, J. L., Kratovac, S., Nelson, D. L., Corbin, J. G., et al. (2013). Homeostatic responses fail to correct defective amygdala inhibitory circuit maturation in fragile $\mathrm{X}$ syndrome. Journal of Neuroscience, 33, 7548-7558.

Vissers, M. E., Cohen, M., \& Geurts, H. M. (2012). Brain connectivity and high functioning autism: A promising path of research that needs refined models, methodological convergence, and stronger behavioral links. Neuroscience \& Biobehavioral Reviews, 36, 604-625.

Voineagu, I., Wang, X., Johnston, P., Lowe, J. K., Tian, Y., Horvath, S., et al. (2011). Transcriptomic analysis of autistic brain reveals convergent molecular pathology. Nature, 474, 380-384.

Vygotsky, L. S. (1991). Genesis of the higher mental functions. Learning to Think, 2, 32 .

Wass, S. V., Scerif, G., \& Johnson, M. H. (2012). Training attentional control and working memory-Is younger, better? Developmental Review, 32, 360-387.

Williams, J. H. G., Waiter, G. D., Gilchrist, A., Perrett, D. I., Murray, A. D., \& Whiten, A. (2006). Neural mechanisms of imitation and "mirror neuron" functioning in autistic spectrum disorder. Neuropsychologia, 44, 610-621.

Willsey, A. J., Sanders, S. J., Li, M., Dong, S., Tebbenkamp, A. T., Muhle, R. A., et al. (2013). Coexpression networks implicate human midfetal deep cortical projection neurons in the pathogenesis of autism. Cell, 155, 9971007.

Wolff, J. J., Gu, H., Gerig, G., Elison, J. T., Styner, M., Gouttard, S., et al. (2012). Differences in white matter fiber tract development present from 6 to 24 months in infants with autism. American Journal of Psychiatry, 169, 589-600.

Yerys, B. E., Wallace, G. L., Sokoloff, J. L., Shook, D. A., James, J. D., \& Kenworthy, L. (2009). Attention deficit/hyperactivity disorder symptoms moderate cognition and behavior in children with autism spectrum disorders. Autism Research, 2, 322-333.

Yizhar, O., Fenno, L. E., Prigge, M., Schneider, F., Davidson, T. J., O'Shea, D. J., et al. (2011). Neocortical excitation/inhibition balance in information processing and social dysfunction. Nature, 477, 171-178.

Zhang, L. I., Bao, S., \& Merzenich, M. M. (2002). Disruption of primary auditory cortex by synchronous auditory inputs during a critical period. Proceedings of the National Academy of Sciences, 99, 2309-2314.

Zikopoulos, B., \& Barbas, H. (2013). Altered neural connectivity in excitatory and inhibitory cortical circuits in autism. Frontiers in Human Neuroscience, 7, 609.

Zoghbi, H. Y. (2003). Postnatal neurodevelopmental disorders: Meeting at the synapse? Science, 302, 826-830. 